\title{
Un nouveau contraceptif parfait... pour le cobaye
}

\section{Les nouvelles de ce numéro ont été préparées par Jean-Claude Dreyfus Jean-Pierre Grünfeld Axel Kahn Marc Peschanski}

Parmi les méthodes contraceptives encore à l'étude, c'est sans doute la vaccination qui est la plus prometteuse. Des essais cliniques sont même en cours [1] avec un vaccin dirigé contre la gonadotrophine chorionique humaine, mais cette méthode garde des inconvénients : outre qu'elle ne met peut-être pas à l'abri des réactions immunologiques secondaires, elle a un mécanisme d'action abortif et non contraceptif, ce qui constitue un handicap éthique important. On comprend donc l'intérêt d'un vaccin susceptible d'interférer avec la reconnaissance sperme-œuf. Or on sait que chez environ $5 \%$ des hommes et des femmes stériles existent des signes d'immunité contre des antigènes spécifiques du sperme sans autre symptôme de morbidité [2]. Cette idée a été exploitée par Primakoff et al. (Farmington, Conn, USA). Ils sont partis [3] du fait qu'ils avaient découvert antérieurement qu'une protéine de surface de spermatozoïdes de cobaye, PH-20, de $64000 \mathrm{Da}$ (daltons), est essentielle pour l'adhérence du spermatozoïde à la couche externe (zone pellucide) de l'œuf. Cette protéine est reconnue par un anticorps monoclonal qui permet de la localiser à la fois sur la membrane plasmique et sur la membrane interne de l'acrosome après son activation. Après « vaccination » avec 10 à $50 \mu \mathrm{g}$ de $\mathrm{PH}-20$, aucun des 25 cobayes femelles immunisées ne fut capable de concevoir, alors que 34 des 36 témoins avaient des portées de trois à quatre petits en moyenne. Un test in vitro révélait que le sérum de ces femelles inhibait la liaison spermatozoïde-zone pellucide. Leur sérum reconnaissait également le $\mathrm{PH}-20$ directement, alors qu'aucune protéine de l'organisme de la femelle ne réagissait, montrant ainsi la spécificité de $\mathrm{PH}-20$ et minimisant tout risque d'auto-anticorps chez la femelle immunisée. Enfin la durée de la stérilité étáit d'environ six mois, avec retour progressif à la normale dans un délai de 15 mois. La méthode cumule donc efficacité parfaite et réversibilité. L'injection aux mâles provoque également la stérilité, réversible dans les mêmes délais, apparemment sans effets secondaires malgré la possibilité théorique de développer des auto-anticorps chez le mâle.

On tient donc, semble-t-il, le vaccin contraceptif idéal, mais c'est... chez le cobaye. Le spermatozoïde humain possède certes des antigènes de surface, qui changent de position lors de l'activation de l'acrosome, mais ils ne ressemblent pas à PH-20. L'impact de l'article de Primakoff et al. devrait être suffisant pour que la recherche des antigènes de surface impliqués dans la reconnaissance spermatozoïde-zone pellucide dans l'espèce humaine en reçoive une impulsion décisive.

J.-C. D.

1. Jones WV, Bradley J, Judd SJ, et al. Phase 1 clinical trial of a world health organization birth control vaccine. Lancet 1988 ; i : 1295-8.

2. Aitken RJ, Paterson M. New horizons in contraception. Nature 1988 ; 335 : 492-3.

3. Primakoff P, Lathrop W, Woolman L, Cowan A, Myles D. Fully effective contraception in male and female guinea pig immunized with the sperm protein PH-20. Nature 1988; 355 : 543-6. 